Preface

\title{
Personalized Approaches to the Treatment of Hemostatic Disorders
}

\author{
Roger J.S. Preston, $\mathrm{PhD}^{1}$ Jamie M. O'Sullivan, $\mathrm{PhD}^{1}$ \\ ${ }^{1}$ Irish Centre for Vascular Biology, School of Pharmacy and \\ Biomolecular Sciences, Royal College of Surgeons in Ireland, Dublin, \\ Ireland \\ Semin Thromb Hemost 2021;47:117-119.
}

The last 20 years has seen dramatic improvements in the clinical management of hemostatic and thrombotic disorders. We have witnessed the introduction of novel therapeutic agents into the clinic, including direct oral anticoagulants (DOACs), and the rapid evolution of personalized therapy for patients with the development of high-throughput genetic analysis and gene therapy. Moreover, growing awareness of nonlinear genotype-phenotype correlations in disease presentation, including bleeding or thrombosis risk, has catalyzed the concept of tailored individualized therapies. Many of these clinical milestones have been made possible by improved understanding of the fundamental biology and thus pathophysiology underpinning hemostatic disorders, as well as development of novel technologies and diagnostic tools. This review series aims to provide an overview of some of these advancements, with a particular focus on novel and personalized approaches to the management of disorders of hemostasis and thrombosis.

The nature of the circulatory system means that regulation of vascular integrity and associated disorders is both complex and dynamic. Hemostatic responses typically occur under conditions of varying shear stress across the vasculature, with platelets flowing across the endothelium and with concentration gradients of specific procoagulant and anticoagulant factors. Studying and understanding the crosstalk between all these processes in in vitro or ex vivo settings presents particular challenges. Nevertheless, the first part of this review series describes the significant advances that have been made in preclinical and diagnostic technologies, including three-dimensional cellularized microfluidic devices, which provide a platform to examine many of these biological components in unison. Caruso and Lam detail the current state-of-the-art technologies underpinning their application in point-of-care diagnostic devices that assess hemostasis parameters in the clinical setting. ${ }^{1}$ Another unique approach

Address for correspondence Jamie O'Sullivan, PhD, Irish Centre for Vascular Biology, School of Pharmacy and Biomolecular Sciences, Royal College of Surgeons in Ireland, 123 St Stephens Green, Dublin 2, Ireland

(e-mail: jamieosullivan@rcsi.ie).

to the challenge of understanding the complex biological interplay regulating hemostasis is computational modeling. In the second contribution of this series, Leiderman et al provide a comprehensive overview of the key inputs in designing robust integrated mathematical models of thrombus formation and how these unique systems can be used to interrogate the complex nature of thrombosis and hemostasis and provide insight into novel disease mechanisms. ${ }^{2}$

In addition to diagnostic and technological advances in this field, the advent of DOACs, first approved for clinical use in 2010, has significantly shifted the therapeutic landscape for venous thromboembolic disease. DOACs have emerged as effective, safe, and convenient therapeutic options for thromboprophylaxis. This is exemplified in amendments to many of the published guidelines, with inclusion of DOACs as therapeutic alternatives for thromboprophylaxis in high-risk cancer patients. In the third part of this issue, Dhami et al summarizes the advances in the management of cancer-associated thrombosis, including insights into the pathophysiology, refinement of risk prediction models, and novel therapies. ${ }^{3}$ This is timely given the recently published data from the much-anticipated CARAVAGGIO trial evaluating the DOAC apixaban in the oncology setting. ${ }^{4}$ The predictable clinical and pharmacokinetic profile of DOACs also favors them as agents of choice over vitamin $\mathrm{K}$ antagonists and low molecular weight heparins for reducing the risk of stroke and for treatment of venous thromboembolism. Revision of international guidelines in the field now places emphasis on a personalized "best-fit" strategy for use of DOACs for thromboprophylaxis..$^{5}$ With the expanding role of DOACs, clinicians are now faced with increasingly complex decisions including duration of treatment and monitoring parameters. The clinical monitoring of plasma DOAC activity in these cohorts can be challenging, given that many routine coagulation assays are not sensitive to DOACs in the same manner as other anticoagulants. ${ }^{6}$ Despite the widespread

(c) 2021. Thieme. All rights reserved. Thieme Medical Publishers, Inc., 333 Seventh Avenue, 18th Floor, New York, NY 10001, USA
DOI https://doi.org/ 10.1055/s-0041-1723800. ISSN 0094-6176.
Approaches to Bleeding and

Thrombotic Disorders; Guest

Editors: Jamie O'Sullivan, PhD and Roger Preston, PhD. 
clinical use of DOACs, currently there are no Food and Drug Administration-approved methods to monitor the anticoagulant effect of DOAC. In this series, Renon et al review the technical aspects of DOAC "monitoring," unique approaches, and examines the clinical applicability of DOAC measurements from trials to real-world evidence. ${ }^{7}$

Genomic analysis and gene therapy are anticipated to be cornerstones of personalized medicine in the modern era. The field of hemostasis, in particular, has experienced very significant advances in the area in recent years with the development of successful gene therapy approaches for hemophilia A and B, which have served as exemplar models of the bench-to-bedside ethos of scientific research. Arruda et al reflects on the gene therapy journey for hemophilia and how these key scientific lessons have now paved the way for development of genetic therapy approaches in other rare inherited bleeding disorders, including factor VII deficiency, von Willebrand disease (VWD), and Glanzmann thrombasthenia. ${ }^{8}$ Furthermore, increased accessibility to high-throughput sequencing technologies has improved our fundamental understanding of the genetic basis underpinning many vascular disorders, allowing for more refined diagnoses, better disease risk assessment, and tailored treatments for patients. ${ }^{9}$ Notwithstanding this, significant challenges remain with the widespread implementation of genetic testing in clinical practice. In this series, Gomez discusses the current sequencing technologies at our disposal and their application in disorders of hemostasis and thrombosis. ${ }^{10}$ He also expertly summarizes how genome-wide association studies have helped shape our understanding of heritability in venous thrombosis.

Improved insights into genotype-phenotype relationships in hemostatic disorders has also fostered personalized therapeutic approaches for the most common inherited bleeding disorders globally, VWD and hemophilia. Several studies report several additional disease modifiers which modulate variability in bleeding phenotype in patients with hemophilia. ${ }^{11}$ Accordingly, prophylactic replacement of missing coagulation factors VIII (FVIII) and IX (FIX) based solely upon endogenous levels may be insufficient to manage bleeding. Rehill et al discusses other biological elements beyond FVIII or FIX levels which significantly influence bleeding phenotype in hemophilia and reflects on individualized therapeutic approaches for the longterm clinical management of these patients. ${ }^{12}$ Similarly, O'Donnell reports on subgroups of patients with partial quantitative VWD, where bleeding risk does not correlate with plasma von Willebrand factor (VWF) levels. ${ }^{13}$ This review highlights the important advances that have been made in our understanding of VWD pathophysiology, including stratification of patients into distinct VWD subgroups, as well as understanding the heterogeneity in response to current therapeutic regimens. Critically, defining optimal therapeutic VWF target levels is of particular clinical importance with respect to designing personalized treatment plans for VWD patients undergoing surgical procedures. The importance of this approach is underscored by the challenges clinicians face in the management of gynecological and obstetric bleeding in pregnant women with VWD, where much clinical uncertainty exists. In support of this, Makhamreh et al reports on a systematic review of pregnancy outcomes, and complications in women with type $2 \mathrm{~B} \mathrm{VWD} .{ }^{14}$ The high frequency of thrombocytopenia in this cohort results in significant morbidity in pregnancy for women with type 2B VWD. Furthermore, Byrne et al discuss the therapeutic approaches and clinical challenges in the management of pregnant women with VWD. ${ }^{14,15}$ Several clinical studies have reported that women with VWD have an increased risk of postpartum hemorrhage. ${ }^{16}$ Despite this, the lack of a significant evidence-based approach means that current management of women with VWD during pregnancy is based on expert consensus guidelines, which are inconsistent internationally. This review provides an in-depth analysis of the unmet clinical needs and current practices in the management of pregnant women with VWD. Taken together, these reviews reflect the urgent requirement for international collaboration and high-quality data to provide a robust evidence basis on which optimal clinical management of these women can be based.

In summary, this issue of Seminars in Thrombosis \& Hemostasis provides a snapshot of several novel and personalized advancements in understanding hemostatic disorders, including unique diagnostic technologies, novel therapeutic avenues, and tailored clinical care for long-term management. However, this progress is not consistent across all fields of hemostasis. In particular, this issue also reflects on the unmet clinical needs of woman with inherited bleeding disorders where further scientific and clinical studies are warranted. We hope this series will be of interest to the readership, including clinicians and scientists alike, and highlight opportunities of further improvements and developments in the future.

\section{Conflict of Interest}

Dr. O'Sullivan reports to receiving research grant funding from LEO Pharma.

\section{References}

1 Caruso C, Lam WA. Point-of-care diagnostic assays and novel preclinical technologies for hemostasis and thrombosis. Semin Thromb Hemost 2021;47(02):120-128

2 Leiderman K, Sindi SS, Monroe DM, Fogelson AL, Neeves KB. The art and science of building a computational model to understand hemostasis. Semin Thromb Hemost 2021;47(02): 129-138

3 Dhami SPS, Patmore S, O'Sullivan JM. Advances in the management of cancer associated thrombosis. Semin Thromb Hemost 2021;47(02):139-149

4 Agnelli G, Becattini C, Meyer G, et al;Caravaggio Investigators. Apixaban for the treatment of venous thromboembolism associated with cancer. N Engl J Med 2020;382(17):1599-1607

5 Khorana AA, Noble S, Lee AYY, et al. Role of direct oral anticoagulants in the treatment of cancer-associated venous thromboembolism: guidance from the SSC of the ISTH. J Thromb Haemost 2018;16(09):1891-1894

6 Gosselin RC, Adcock DM, Bates SM, et al. International Council for Standardization in Haematology (ICSH) recommendations for laboratory measurement of direct oral anticoagulants. Thromb Haemost 2018;118(03):437-450

7 Renon F, Rago A, Liccardo B, et al. Direct oral anticoagulants plasma levels measurement: clinical usefulness from trials to real-world data. Semin Thromb Hemost 2021;47(02): $150-160$ 
8 Arruda VR, Weber J, Samelson-Jones BJ. Gene therapy for inherited bleeding disorders. Semin Thromb Hemost 2021;47(02): 161-173

9 Downes K, Megy K, Duarte D, et al;NIHR BioResource. Diagnostic high-throughput sequencing of 2396 patients with bleeding, thrombotic, and platelet disorders. Blood 2019;134(23):2082-2091

10 Gomez K. Genomic analysis for the detection of bleeding and thrombotic disorders. Semin Thromb Hemost 2021;47(02): 174-182

11 Carcao MD, van den Berg HM, Ljung R, Mancuso MEPedNet and the Rodin Study Group. Correlation between phenotype and genotype in a large unselected cohort of children with severe hemophilia A. Blood 2013;121(19):3946-3952, S1

12 Rehill AM, McCluskey S, O'Donnell JS, Dockal M, Preston RJS. Heterogeneity in bleeding tendency and arthropathy develop- ment in individuals with haemophilia. Semin Thromb Hemost 2021;47(02):183-191

13 O'Donnell JS. Towards personalized treatment for patients with Low VWF and quantitative von Willebrand disease. Semin Thromb Hemost 2021;47(02):192-200

14 Makhamreh MM, Russo ML, Karl T, Delgado N, Lackritz K, Skupski DW, Al-Kouatly HB. Type 2B von Willebrand disease in pregnancy: a systematic literature review. Semin Thromb Hemost 2021;47 (02):201-216

15 Byrne B, Ryan K, Lavin M. Current challenges in the peripartum management of women with von Willebrand Disease. Semin Thromb Hemost 2021;47(02):217-228

16 Lavin M, Aguila S, Dalton N, et al. Significant gynecological bleeding in women with low von Willebrand factor levels. Blood Adv 2018;2(14):1784-1791 